Article

\title{
Biological Activities of Selected Plants and Detection of Bioactive Compounds from Ardisia elliptica Using UHPLC-Q-Exactive Orbitrap Mass Spectrometry
}

\author{
Pei Lou Wong ${ }^{1}$, Nurul Azila Fauzi ${ }^{1}$, Siti Norhamimah Mohamed Yunus ${ }^{1}$, \\ Nur Ashikin Abdul Hamid ${ }^{2}$, Siti Zulaikha Abd Ghafar ${ }^{1}$, Awanis Azizan ${ }^{2}$, \\ Nur Khaleeda Zulaikha Zolkeflee ${ }^{2}$ and Faridah Abas $1,2, *$ iD \\ 1 Department of Food Science, Faculty of Food Science and Technology, Universiti Putra Malaysia, \\ Serdang 43400, Selangor, Malaysia; plou_wong@hotmail.com (P.L.W.); azilafauzi9520@gmail.com (N.A.F.); \\ cikmiemayunus@gmail.com (S.N.M.Y.); ctzue.agb@gmail.com (S.Z.A.G.) \\ 2 Laboratory of Natural Products, Institute of Bioscience, Universiti Putra Malaysia, Serdang 43400, Selangor, \\ Malaysia; shikinhamid89@yahoo.com (N.A.A.H.); awanis_azizan@yahoo.com (A.A.); \\ khaleeda_zulaikha@yahoo.com (N.K.Z.Z.) \\ * Correspondence: faridah_abas@upm.edu.my; Tel.: +60-397-698-343; Fax: +60-389-423-552
}

Academic Editor: Roberto Fabiani

Received: 1 May 2020; Accepted: 4 June 2020; Published: 6 July 2020

\begin{abstract}
Plants and plant-based products have been used for a long time for medicinal purposes. This study aimed to determine the antioxidant and anti- $\alpha$-glucosidase activities of eight selected underutilized plants in Malaysia: Leucaena leucocephala, Muntingia calabura, Spondias dulcis, Annona squamosa, Ardisia elliptica, Cynometra cauliflora, Ficus auriculata, and Averrhoa bilimbi. This study showed that the $70 \%$ ethanolic extract of all plants exhibited total phenolic content (TPC) ranging from 51 to $344 \mathrm{mg}$ gallic acid equivalent (GAE)/g dry weight. A. elliptica showed strong 2,2-diphenyl-1-picrylhydrazyl (DPPH) and nitric oxide (NO) scavenging activities, with half maximal inhibitory concentration $\left(\mathrm{IC}_{50}\right)$ values of 2.17 and $49.43 \mu \mathrm{g} / \mathrm{mL}$, respectively. Most of the tested plant extracts showed higher inhibition of $\alpha$-glucosidase enzyme activity than the standard, quercetin, particularly A. elliptica, F. auriculata, and M. calabura extracts with $\mathrm{IC}_{50}$ values of 0.29 , 0.36 , and $0.51 \mu \mathrm{g} / \mathrm{mL}$, respectively. A total of 62 metabolites including flavonoids, triterpenoids, benzoquinones, and fatty acids were tentatively identified in the most active plant, i.e., A. elliptica leaf extract, by using ultra-high-performance liquid chromatography (UHPLC)-electrospray ionization (ESI) Orbitrap MS. This study suggests a potential natural source of antioxidant and $\alpha$-glucosidase inhibitors from $A$. elliptica.
\end{abstract}

Keywords: underutilized plants; antioxidant; free radical scavenging; anti- $\alpha$-glucosidase; phytochemical characterization

\section{Introduction}

Malaysia is a country that is recognized for its diverse flora and fauna. Various species of plants, animals, and microorganisms offer Malaysians an extensive source of nutritious food and medicines. Furthermore, the antioxidant activities of different parts of plants, including roots, leaves, stalks, flowers, fruits, and seeds were studied. Acknowledgement of the potential medicinal benefits of local plants along with the development of modern technology motivated researchers, pharmacists, and physicians to explore Malaysian biodiversity. In addition to commonly consumed local herbs and fruits, some underutilized local species have the potential to act as alternative sources of micronutrients, vitamins, and health-promoting secondary plant metabolites [1]. These species include "petai belalang" (Leucaena leucocephala (Lam.) de Wit (Fabaceae)), "ceri hutan" (Muntingia calabura L. (Muntingiaceae)), 
"kedondong" (Spondias dulcis Parkinson (Anacardiaceae)), "nona" (Annona squamosal L. (Annonaceae)), "mata ayam" (Ardisia elliptica Thunb. (Primulaceae)), "katak puru" (Cynometra cauliflora L. (Fabaceae)), "ara" (Ficus auriculata Lour. (Moraceae)), "belimbing buluh" (Averrhoa bilimbi L. (Oxalidaceae)), and others. However, the biological activity and the chemical profile of these underutilized plants remain unknown. Therefore, this study was conducted to fill in the current research gap existing for these plants.

The prevalence of Malaysian adults suffering from diabetes mellitus increased from 11.6\% in 2006 to $15.2 \%$ in 2011 ; the rate is projected to reach $21.6 \%$ by 2020 [2]. Previous studies showed correlations between oxidative stress and diabetes [3]. Human bodies rely on endogenous and exogenous antioxidants to minimize the cellular damage and stress caused by free radicals by maintaining redox balance. Bouayed and Bohn [4] stated that antioxidants from fruits, vegetables, and other sources play a significant role in assisting the endogenous antioxidant defense system, which includes superoxide dismutase, catalase, and glutathione peroxidase, in preventing oxidative stress.

Diabetic patients suffer from an abnormal increase of blood glucose level after meal ingestion, a condition commonly known as postprandial hyperglycemia. $\alpha$-Glucosidase, which is located in the epithelium of the small intestine, is one of the enzymes responsible for carbohydrate digestion. Postprandial hyperglycemia can be reduced through several means such as by suppressing $\alpha$-glucosidase activity, thereby delaying the carbohydrate hydrolysis and glucose absorption by the cells [5]. Triggle and Ding [6] reported that synthetic drugs, such as metformin, sulfonylureas, thiazolidinediones, and other $\alpha$-glucosidase inhibitors (including acarbose and miglitol, which were introduced as treatment for diabetes and are also known for their undesirable side effects) increased cardiovascular risk and induction of hepatotoxicity. Since modern medical treatments encourage the use of plant-based functional foods and drugs, particularly in diabetes treatment, numerous studies were conducted in the quest for effective hypoglycemic agents. Kumar et al. [7] suggested that natural $\alpha$-glucosidase inhibitors from plant sources, including flavonoids, alkaloids, terpenoids, anthocyanins, glycosides, and phenolic compounds, are effective in inhibiting the activity of $\alpha$-glucosidase. Therefore, this study aimed to determine the total phenolic content (TPC), as well as antioxidant (2,2-diphenyl-1-picrylhydrazyl (DPPH) and nitric oxide (NO) free radical scavenging) and anti- $\alpha$-glucosidase activities, of the leaves of selected underutilized Malaysian plants. This study provides the first detailed metabolite profile of the most active extract, i.e., Ardisia elliptica, by using ultra-high-performance liquid chromatography (UHPLC)-electrospray ionization (ESI) Orbitrap MS.

\section{Results and Discussion}

\subsection{Total Phenolic Content (TPC) of the Selected Plant Extracts}

The current study showed that all leaf extracts had high TPC concentrations ranging from $50.90 \pm 0.69$ to $344.17 \pm 10.80 \mathrm{mg}$ gallic acid equivalent (GAE)/g crude extract (Table 1). The leaf extract of $C$. cauliflora had the highest phenolic content, followed by that of A. elliptica and A. squamosa $(253.10 \pm 1.19$ and $199.62 \pm 7.40 \mathrm{mg} \mathrm{GAE} / \mathrm{g}$ crude extract, respectively), while the leaf extract of S. dulcis had the lowest phenolic content. A lower TPC value for S. dulcis was also reported by Rahman et al. [8]. Unlike other species from the Spondias family, this particular species was not thoroughly studied, probably due to its low phenolic content. The TPCs of L. leucocephala, M. calabura, and F. auriculata were not significantly different $(p>0.05)$, with values of $175.75 \pm 3.48,172.32 \pm 3.39$, and $167.15 \pm 2.04 \mathrm{mg}$ GAE/g crude extract, respectively, followed by the leaf extract of $A$. bilimbi at $97.50 \pm 3.46 \mathrm{mg}$ GAE/g crude extract. Variations in the applied extraction system might influence the phenolic content evaluated in plant extracts. Ethanol was believed to be able to extract more phenolic compounds compared to acetone, water, and methanol [9]. Methanolic A. squamosa and C. cauliflora leaf extracts were reported to have lower TPC compared to current study [10,11], while the $50 \%$ ethanolic M. calabura extract was found to retain higher TPC compared to absolute ethanol and water extracts [12]. Meanwhile, soaking of $A$. elliptica leaves in 95\% methanol yielded a lower TPC 
compared to the present study which employed sonication-assisted extraction [13]. Furthermore, soaking of $A$. bilimbi leaves in $70 \%$ ethanol was found to result in higher TPC compared to the current extract [14]. Despite the choice of organic solvents used and the water content present in the extraction, the level of phenolic compounds produced in plant tissue might be affected by environmental factors, climatic factors, and soil nutrients [15].

Table 1. Total phenolic content (TPC), 2,2-diphenyl-1-picrylhydrazyl (DPPH) and nitric oxide (NO) free radical scavenging, and anti- $\alpha$-glucosidase activities of the extracts. $\mathrm{IC}_{50}$-half maximal inhibitory concentration; GAE-gallic acid equivalent.

\begin{tabular}{|c|c|c|c|c|}
\hline \multirow{2}{*}{$\begin{array}{c}\text { Leaves } \\
\text { Extracts/Standard }\end{array}$} & \multirow{2}{*}{$\begin{array}{l}\text { Total Phenolic Content } \\
\text { (mg GAE/g Sample) }\end{array}$} & \multicolumn{3}{|c|}{$\mathrm{IC}_{50}$ Value $(\mu \mathrm{g} / \mathrm{mL})$} \\
\hline & & $\begin{array}{l}\text { DPPH Free Radical } \\
\text { Scavenging Activity }\end{array}$ & $\begin{array}{l}\text { NO Free Radical } \\
\text { Scavenging Activity }\end{array}$ & $\begin{array}{c}\text { Anti- } \alpha \text {-Glucosidase } \\
\text { Activity }\end{array}$ \\
\hline Leucaena leucocephala & $175.75 \pm 3.48^{\mathrm{d}}$ & $8.67 \pm 0.29^{d}$ & $65.57 \pm 4.57^{b}$ & $6.62 \pm 0.19^{c}$ \\
\hline Muntingia calabura & $172.32 \pm 3.39^{d}$ & $4.67 \pm 0.21^{c}$ & $59.40 \pm 3.39^{b}$ & $0.51 \pm 0.01^{\mathrm{a}}$ \\
\hline Spondias dulcis & $50.90 \pm 0.69^{f}$ & $14.22 \pm 0.82^{\mathrm{e}}$ & $301.66 \pm 23.06^{f}$ & $45.52 \pm 2.18^{e}$ \\
\hline Annona squamosa & $199.62 \pm 7.40^{c}$ & $5.00 \pm 0.20^{c}$ & $109.02 \pm 3.18^{c}$ & $3.59 \pm 0.18^{b}$ \\
\hline Ardisia elliptica & $253.10 \pm 1.19^{b}$ & $2.17 \pm 0.08^{\mathrm{a}}$ & $49.43 \pm 0.18^{b}$ & $0.29 \pm 0.01^{\mathrm{a}}$ \\
\hline Cynometra cauliflora & $344.17 \pm 10.80^{\mathrm{a}}$ & $2.88 \pm 0.05^{a b}$ & $118.62 \pm 3.44^{\mathrm{cd}}$ & $0.90 \pm 0.02^{\mathrm{a}}$ \\
\hline Ficus auriculata & $167.15 \pm 2.04^{\mathrm{d}}$ & $5.06 \pm 0.35^{c}$ & $169.65 \pm 1.53^{\mathrm{e}}$ & $0.36 \pm 0.02^{\mathrm{a}}$ \\
\hline Averrhoa bilimbi & $97.50 \pm 3.46^{\mathrm{e}}$ & $16.80 \pm 0.04^{\mathrm{f}}$ & $134.33 \pm 2.46^{\mathrm{d}}$ & $26.91 \pm 0.58^{d}$ \\
\hline Quercetin & - & $3.55 \pm 0.28^{b}$ & $15.85 \pm 0.58^{a}$ & $6.62 \pm 0.03^{c}$ \\
\hline Gallic acid & - & - & $15.41 \pm 0.63^{a}$ & - \\
\hline
\end{tabular}

The results are expressed as means \pm standard deviation. Means with different superscript letters are significantly different $(p<0.05)$ among leaf extracts. "_" indicates that the particular activities were not measured because of irrelevance to the compound.

\subsection{DPPH and NO Free Radical Scavenging Activity of the Selected Plant Extracts}

Two in vitro antioxidant assays were used to investigate the antioxidant potential of selected leaf extracts, which using DPPH and NO free radical scavenging assays. A. elliptica and C. cauliflora leaf extracts showed stronger antioxidant capability than the standard quercetin in the DPPH assay, whereas none of the leaf extracts showed higher activity than the standards quercetin and gallic acid in the NO assay. In addition, no similar trend was observed in free radical scavenging activities when the DPPH and NO assays were compared (Table 1). A. elliptica, C. cauliflora, and M. calabura extracts showed high activity in DPPH assay with half maximal inhibitory concentration $\left(\mathrm{IC}_{50}\right)$ values of $2.17 \pm 0.08$, $2.88 \pm 0.05$, and $4.67 \pm 0.21 \mu \mathrm{g} / \mathrm{mL}$, respectively. On the other hand, the plant extracts that showed strong capability in scavenging $\mathrm{NO}$ radicals were $A$. elliptica and $M$. calabura extracts, with $\mathrm{IC}_{50}$ values of $49.43 \pm 0.18$ and $59.40 \pm 3.39 \mu \mathrm{g} / \mathrm{mL}$, respectively. However, C. cauliflora showed weak inhibition in the $\mathrm{NO}$ assay, with an $\mathrm{IC}_{50}$ value of $118.62 \pm 3.44 \mu \mathrm{g} / \mathrm{mL}$. This might be due to the slight difference in mechanism between both assays. DPPH radicals were scavenged by antioxidants that act as a hydrogen donor [16]. On the other hand, two possible pathways are available to scavenge NO radicals: one is the removal of hydrogen from antioxidants, and the other is by receiving a single-electron transfer from the NO radical to form an antioxidant cation, followed by an oxidation process [17].

Despite the divergence of mechanism of both antioxidant assays, the current study showed that $A$. elliptica leaf extract had the greatest antioxidant potential among all tested plants in the DPPH and NO scavenging assays, with $\mathrm{IC}_{50}$ values of $2.17 \pm 0.08$ and $49.43 \pm 0.18 \mu \mathrm{g} / \mathrm{mL}$, respectively (Table 1 ). Pearson correlation was employed to evaluate the association between TPC and antioxidant activities of the active extracts. A. elliptica and C. cauliflora demonstrated week and moderate positive correlation between TPC and the DPPH assay with $R$ values of 0.27 and 0.46 , respectively. $M$. calabura showed strong positive correlation $(R$ value $=0.87$, which suggests that phenolic compounds in $M$. calabura contribute to the DPPH assay as reported by previous study [12]. Furthermore, it is worth to note that C. cauliflora demonstrated a strong negative correlation with $R$ value $=-0.99$ between TPC and the NO assay. Other types of metabolites such as tannin, terpenoids, and saponins found in $C$. cauliflora leaves might provide a positive effect on the bioactivities [18]. In addition, A. elliptica showed a strong positive correlation with $R$ value $=0.87$ between TPC and the NO assay, implying that secondary metabolites 
as phenolic compounds may be responsible for the plants' antioxidant capability, which supported the corresponding high TPC values in A. elliptica leaf extract [4,19].

\subsection{Anti- $\alpha$-Glucosidase Activity of the Selected Plant Extracts}

Among the tested plant samples, $S$. dulcis showed the weakest anti- $\alpha$-glucosidase activity followed by $A$. bilimbi, with $\mathrm{IC}_{50}$ values of $45.52 \pm 2.18$ and $26.91 \pm 0.58 \mu \mathrm{g} / \mathrm{mL}$, respectively (Table 1 ). The remaining leaf extracts had potent enzyme inhibition activity with $\mathrm{IC}_{50}$ values between $0.29 \pm 0.01$ and $6.62 \pm 0.19 \mu \mathrm{g} / \mathrm{mL}$. The $\mathrm{IC}_{50}$ values shown by most of the leaf extracts were equal to or lower than that of the positive control, quercetin, suggesting the potential for the use of plant-based material as an anti-diabetic agent. A. elliptica, F. auriculata, M. calabura, and C. cauliflora leaf extracts showed high activity for the anti- $\alpha$-glucosidase enzymatic reaction without a statistical difference $(p>0.05)$, with $\mathrm{IC}_{50}$ values ranging from $0.29 \pm 0.01$ to $0.90 \pm 0.02 \mu \mathrm{g} / \mathrm{mL}$. Pearson correlation analysis showed that the $C$. cauliflora extract demonstrated strong positive correlation between TPC and anti- $\alpha$-glucosidase activity $(R=0.84)$, while A. elliptica and $M$. calabura extracts showed moderate positive correlation with $R$ values of 0.71 and 0.59 , respectively. The high content of phenolic compounds could contribute to the activity of these plants owing to the notable high TPC observed in the current study [20]. Apart from phenolic compounds, the presence of plant steroids, triterpenoids, and other nitrogenous compounds was also responsible for anti-diabetic effects [7]. The complexity of plant metabolites anticipated the simultaneous interactions of various phytoconstituents either synergistically or antagonistically on the bioactivities [21]. Therefore, the findings of this study further strengthen previous claims regarding the anti-diabetic potential of these plants.

\subsection{UHPLC-MS/MS Analysis of Ardisia Elliptica}

Based on biological activity results, A. elliptica was identified as the most potent extract that showed antioxidant and anti- $\alpha$-glucosidase activities. In view of the limited published information regarding the metabolite profile, further phytochemical characterization of the active extract was performed by using UPLC-ESI-Orbitrap MS/MS. A total of 62 metabolites were tentatively characterized in A. elliptica leaf extract (Table 2). The identification of the metabolites was based on the comparison with the literature and several online mass databases (Knapsack, Metabolomics Workbench, Human Metabolome Database, PubChem, and MassBank). The total ion chromatogram showed the major components observed between 0 and $30 \mathrm{~min}$ (Figure 1). Flavonoids accounted for $63 \%$ of the 62 tentatively identified metabolites, which may contribute to the high TPC value of $A$. elliptica leaf extract. Flavonoids such as quercetin, kaempferol, myricetin, and catechin derivatives, which are known to be abundant in plant matrices, could be identified in A. elliptica leaf extract. Other types of metabolites identified include fatty acid derivatives, benzoquinones, triterpenoids, phenolic lipids, and phenol ester. 
Table 2. Mass spectral characteristics and tentative identification of compounds present in $70 \%$ ethanolic leaves extract of $A$. elliptica.

\begin{tabular}{|c|c|c|c|c|c|c|c|}
\hline Peak No. & $\begin{array}{l}\text { Retention } \\
\text { Time, Min }\end{array}$ & Exact Mass & $\begin{array}{l}\text { Deprotonated Molecule } \\
{[\mathrm{M}-\mathrm{H}]^{-}(\mathrm{m} / \mathrm{z})}\end{array}$ & Delta & MS/MS Fragment Ions & Tentative Identification & Molecular Formula \\
\hline 1 & 1.00 & 192.0197 & 191.0184 & 0.0013 & $173.0080,129.0180,111.0074,87.0073$ & Citric acid & $\mathrm{C}_{6} \mathrm{H}_{8} \mathrm{O}_{7}$ \\
\hline 2 & 1.19 & 332.0671 & 331.0662 & 0.0009 & $312.1069,271.0454,241.0342,211.0238,169.0130$ & Monogalloylglucose & $\mathrm{C}_{13} \mathrm{H}_{16} \mathrm{O}_{10}$ \\
\hline 3 & 3.14 & 452.4087 & 451.3394 & 0.0693 & $302.5710,289.0708,210.2854,151.2638$ & (+)-Catechin 6-C-glucoside & $\mathrm{C}_{21} \mathrm{H}_{24} \mathrm{O}_{11}$ \\
\hline 4 & 3.31 & 282.0817 & 281.0331 & 0.0486 & $239.1548,219.1387,207.1384,201.1277,165.0904$ & 5,7-Dimethoxyflavone & $\mathrm{C}_{17} \mathrm{H}_{14} \mathrm{O}_{4}$ \\
\hline 5 & 3.40 & 282.0817 & 281.0329 & 0.0488 & $239.1548,219.1387,207.1384,201.1277,165.0904$ & 5,7-Dimethoxyflavone isomer & $\mathrm{C}_{17} \mathrm{H}_{14} \mathrm{O}_{4}$ \\
\hline 6 & 3.45 & 282.0817 & 281.0328 & 0.0489 & $239.1548,219.1387,207.1384,201.1277,165.0904$ & 5,7-Dimethoxyflavone isomer & $\mathrm{C}_{17} \mathrm{H}_{14} \mathrm{O}_{4}$ \\
\hline 7 & 3.70 & 762.1571 & 761.1343 & 0.0228 & $610.1257,301.0352,169.0131$ & Quercetin 3-O-(2"-O-galloyl)-rutinoside & $\mathrm{C}_{34} \mathrm{H}_{34} \mathrm{O}_{20}$ \\
\hline 8 & 3.80 & 550.1402 & 549.1448 & 0.0046 & $429.1028,369.0819,339.0715,309.0611,269.0662$ & Formononetin 7-O-(2"-p-hydroxybenzoylglucoside) & $\mathrm{C}_{29} \mathrm{H}_{26} \mathrm{O}_{11}$ \\
\hline 9 & 4.14 & 463.1168 & $461.1610^{*}$ & 0.0442 & $314.0427,300.1083,287.0562,255.0286$ & Oxycoccicyanin/Peonidin-3-glucoside & $\mathrm{C}_{22} \mathrm{H}_{23} \mathrm{O}_{11}$ \\
\hline 10 & 4.34 & 454.1555 & 453.1605 & 0.0050 & $386.9793,367.0700,301.0338,284.0323,176.0435$ & 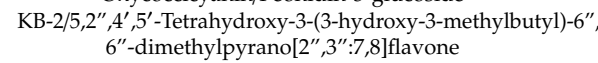 & $", \quad \mathrm{C}_{25} \mathrm{H}_{26} \mathrm{O}_{8}$ \\
\hline 11 & 4.57 & 914.1469 & 913.1451 & 0.0018 & $761.1329,743.1249,609.1331,591.1135,575.0852,453.0853$ & Theasinensin $\mathrm{A}$ & $\mathrm{C}_{44} \mathrm{H}_{34} \mathrm{O}_{22}$ \\
\hline 12 & 4.79 & 350.0024 & 349.0591 & 0.0567 & $269.6351,241.0011,227.0375,152.0433$ & Apigenin 7-sulfate & $\mathrm{C}_{15} \mathrm{H}_{10} \mathrm{O}_{8} \mathrm{~S}$ \\
\hline 13 & 4.95 & 290.0790 & 289.1802 & 0.1012 & $245.0812,203.0703,179.0335,137.0230,123.0437$ & Catechin & $\mathrm{C}_{15} \mathrm{H}_{14} \mathrm{O}_{6}$ \\
\hline 14 & 5.02 & 458.0776 & 457.0766 & 0.0010 & $331.0454,305.0666,287.0562,269.0456,193.0132,169.0131$ & (-)-epigallocatechin-3-gallate & $\mathrm{C}_{22} \mathrm{H}_{18} \mathrm{O}_{11}$ \\
\hline 15 & 5.61 & 756.2040 & 755.2025 & 0.0015 & $489.1044,301.0315,300.0270,271.0243,255.0294,178.9978$ & Quercetin 3-O-(2,6-di-O-rhamnosylglucoside) & $\mathrm{C}_{33} \mathrm{H}_{40} \mathrm{O}_{20}$ \\
\hline 16 & 5.71 & 480.0831 & 479.0816 & 0.0015 & $316.0219,287.0189,271.0241,178.9979,151.0025$ & Myricetin-3-O-glucoside & $\mathrm{C}_{21} \mathrm{H}_{20} \mathrm{O}_{13}$ \\
\hline 17 & 5.76 & 626.1410 & 625.1396 & 0.0014 & $478.0751,317.0288,316.0212,271.0243,178.9976$ & Myricetin-3-O-rutinoside & $\mathrm{C}_{27} \mathrm{H}_{30} \mathrm{O}_{17}$ \\
\hline 18 & 5.93 & 596.1305 & 595.1293 & 0.0012 & $463.0802,301.0349,300.0271,283.0230,271.0244,178.9975$ & Quercetin 3-lathyroside & $\mathrm{C}_{26} \mathrm{H}_{28} \mathrm{O}_{16}$ \\
\hline 19 & 6.11 & 450.0726 & 449.0713 & 0.0013 & $316.0220,287.0198,271.0241,178.9975$ & Myricetin-3-O-arabinoside & $\mathrm{C}_{20} \mathrm{H}_{18} \mathrm{O}_{12}$ \\
\hline 20 & 6.32 & 610.1461 & 609.1451 & 0.0010 & $463.0797,447.0925,301.0349,300.0247$ & Quercetin 3-O- $\alpha$-L-rhamnoside-7-O- $\beta$-D-glucoside & $\mathrm{C}_{27} \mathrm{H}_{30} \mathrm{O}_{16}$ \\
\hline 21 & 6.38 & 610.1461 & 609.1450 & 0.0011 & $301.0339,300.0269,271.0244,178.9973,151.0026$ & Quercetin-3-O-rutinoside (Rutin) & $\mathrm{C}_{27} \mathrm{H}_{30} \mathrm{O}_{16}$ \\
\hline 22 & 6.45 & 464.0882 & 463.0869 & 0.0013 & $316.0218,287.0193,178.9977,151.0026$ & Myricetin-3-O-rhamnoside & $\mathrm{C}_{21} \mathrm{H}_{20} \mathrm{O}_{12}$ \\
\hline 23 & 6.47 & 381.9922 & 380.9911 & 0.0011 & $301.0349,300.0247,283.0245,271.0238,257.0445,229.0496,193.0133$ & Quercetin 3-O-sulfate & $\mathrm{C}_{15} \mathrm{H}_{10} \mathrm{O}_{10} \mathrm{~S}$ \\
\hline 24 & 6.66 & 464.0882 & 463.0875 & 0.0007 & $301.0339,300.0270,271.0244,255.0293,178.9976,151.0024$ & Quercetin-3-O-glucoside & $\mathrm{C}_{21} \mathrm{H}_{20} \mathrm{O}_{12}$ \\
\hline 25 & 6.95 & 434.0776 & 433.0768 & 0.0008 & $301.0346,300.0271,271.0243,255.0291,178.9982,151.0023$ & Quercetin-3-O-arabinoside & $\mathrm{C}_{20} \mathrm{H}_{18} \mathrm{O}_{11}$ \\
\hline 26 & 7.18 & 286.0405 & 285.0395 & 0.0012 & $257.0450,213.0545,187.0391,163.0021$ & Kaempferol & $\mathrm{C}_{15} \mathrm{H}_{10} \mathrm{O}_{6}$ \\
\hline 27 & 7.19 & 365.9973 & 364.9961 & 0.0010 & $\begin{array}{c}285.0400,267.0294,255.0291,241.0501,229.0500,213.0548,178.4121 \\
133.0279,105.6355\end{array}$ & Luteolin 7-sulfate & $\mathrm{C}_{15} \mathrm{H}_{10} \mathrm{O}_{9} \mathrm{~S}$ \\
\hline 28 & 7.44 & 448.0933 & 447.0917 & 0.0016 & $301.0339,300.0271,271.0243,255.0291,178.9975,151.0024$ & Quercetin-3-O-rhamnoside & $\mathrm{C}_{21} \mathrm{H}_{20} \mathrm{O}_{11}$ \\
\hline 29 & 7.64 & 416.1035 & 415.1962 & 0.0927 & $252.1096,238.9105,177.2131,123.0804$ & 7,2'-Dihydroxyflavone 7-glucoside & $\mathrm{C}_{21} \mathrm{H}_{20} \mathrm{O}_{9}$ \\
\hline 30 & 8.31 & 432.0984 & 431.0970 & 0.0014 & $285.0393,284.0321,255.0293,227.0341$ & Kaempferol-3-O-rhamnoside & $\mathrm{C}_{21} \mathrm{H}_{20} \mathrm{O}_{10}$ \\
\hline 31 & 8.66 & 458.0776 & 457.0766 & 0.0010 & $331.0454,305.0666,287.0562,269.0456,193.0132,169.0131$ & Epigallocatechin-3-gallate isomer & $\mathrm{C}_{22} \mathrm{H}_{18} \mathrm{O}_{11}$ \\
\hline 32 & $\begin{array}{l}0.00 \\
9.15\end{array}$ & 396.0079 & 395.0064 & 0.0015 & $\begin{array}{l}315.0607,272.0317,259.0608,151.0027 \\
3.000100\end{array}$ & Persicarin//sorhamnetin 3-sulfate & $\mathrm{C}_{16}^{22} \mathrm{H}_{12} \mathrm{O}_{10} \mathrm{~S}$ \\
\hline 33 & 9.57 & 302.0354 & 301.0348 & 0.0006 & $273.0403,178.9974,151.0024,121.0281$ & Quercetin & $\mathrm{C}_{15} \mathrm{H}_{10} \mathrm{O}_{7}$ \\
\hline 34 & 10.00 & 380.0129 & 379.0117 & 0.0012 & $\begin{array}{c}299.0555,284.0321,257.0403,243.0656,228.0399,211.0385,162.5436 \\
151.0027,110.0001\end{array}$ & Rhamnocitrin 3-O-sulfate & $\mathrm{C}_{16} \mathrm{H}_{12} \mathrm{O}_{9} \mathrm{~S}$ \\
\hline 35 & 10.37 & 542.0658 & 541.0644 & 0.0014 & $461.1082,314.0426,299.0188,271.0243,256.0363,158.7938$ & Isoscutellarein $4^{\prime}$-methyl ether 8 -(2'-sulfatoglucoside) & $\mathrm{C}_{22} \mathrm{H}_{22} \mathrm{O}_{14} \mathrm{~S}$ \\
\hline 36 & 11.20 & 328.2177 & 327.2171 & 0.0006 & $229.1440,211.1331,171.1015$ & Trihydroxy octadecadienoic acid & $\mathrm{C}_{18} \mathrm{H}_{32} \mathrm{O}_{5}$ \\
\hline 37 & 11.98 & 328.2177 & 327.2171 & 0.0006 & $229.1440,211.1331,171.1015$ & Trihydroxy octadecadienoic acid isomer & $\mathrm{C}_{18} \mathrm{H}_{32} \mathrm{O}_{5}$ \\
\hline 38 & 13.78 & 376.2541 & 375.1778 & 0.0763 & $333.6364,330.1770,329.1730,307.1919,235.1334,207.0993$ & Ardisiphenol B & $\mathrm{C}_{23} \mathrm{H}_{36} \mathrm{O}_{4}$ \\
\hline 39 & 14.49 & 290.2173 & 289.1803 & 0.0370 & $245.1902,161.9148,148.7701,123.0794$ & Ardisinol II & $\mathrm{C}_{19} \mathrm{H}_{30} \mathrm{O}_{2}$ \\
\hline 40 & 16.00 & 884.5061 & 883.4165 & 0.0896 & $837.4141,559.1864,456.2514,397.1332,277.2172$ & Ardisianoside D & $\mathrm{C}_{46} \mathrm{H}_{76} \mathrm{O}_{16}$ \\
\hline 41 & 16.37 & 346.2497 & 345.1830 & 0.0667 & $\begin{array}{l}306.9802,292.2949,192.5377 \\
\end{array}$ & Ardisianone A & $\mathrm{C}_{22} \mathrm{H}_{34} \mathrm{O}_{3}$ \\
\hline 42 & 16.54 & 722.1410 & 721.3632 & 0.2222 & $675.3585,569.1615,415.1444,400.9850,305.0875,277.2165$ & Thonningianin $B$ & $\mathrm{C}_{35} \mathrm{H}_{30} \mathrm{O}_{17}$ \\
\hline 43 & 16.96 & 722.1410 & 721.3631 & 0.2221 & $675.3580,569.1614,415.1447,400.9853,305.0875,277.2166$ & Thonningianin $B$ isomer & $\mathrm{C}_{35} \mathrm{H}_{30} \mathrm{O}_{17}$ \\
\hline 44 & 18.14 & 294.1758 & 293.2114 & 0.0356 & 275.2011, 155.1072, 141.1270, 127.1115, 121. 1009 & Embelin & $\mathrm{C}_{17} \mathrm{H}_{26} \mathrm{O}_{4}$ \\
\hline 45 & 18.67 & 560.0399 & 559.1269 & 0.0870 & $354.6981,286.8783,228.8837,121.6282$ & Gossypetin 8-glucoside-3-sulfate & $\mathrm{C}_{21} \mathrm{H}_{20} \mathrm{O}_{16} \mathrm{~S}$ \\
\hline 46 & 19.33 & 426.3789 & 425.2304 & 0.1485 & $271.0612,245.0811,203.0705,177.0179,151.0386$ & Friedelan-3-one & $\mathrm{C}_{30} \mathrm{H}_{50} \mathrm{O}$ \\
\hline 47 & 20.00 & 572.2621 & 571.2880 & 0.0259 & $530.8586,487.1684,391.2236,255.2324,241.0111,223.0012$ & Ardisiaquinone G & $\mathrm{C}_{31} \mathrm{H}_{40} \mathrm{O}_{10}$ \\
\hline 48 & 22.76 & 378.2334 & 377.2329 & 0.0005 & $\begin{array}{c}35.00000,222,335.2515,316.2326,152.0106 \\
359\end{array}$ & Cornudentanone & $\mathrm{C}_{22} \mathrm{H}_{34} \mathrm{O}_{5}$ \\
\hline
\end{tabular}


Table 2. Cont.

\begin{tabular}{|c|c|c|c|c|c|c|c|}
\hline Peak No. & $\begin{array}{l}\text { Retention } \\
\text { Time, Min }\end{array}$ & Exact Mass & $\begin{array}{l}\text { Deprotonated Molecule } \\
{[\mathrm{M}-\mathrm{H}]^{-}(\mathrm{m} / \mathrm{z})}\end{array}$ & Delta & MS/MS Fragment Ions & Tentative Identification & Molecular Formula \\
\hline 49 & 23.12 & 324.0328 & 323.1162 & 0.0834 & $279.2323,265.8335,216.0093,184.0194$ & 6-Chlorocatechin & $\mathrm{C}_{15} \mathrm{H}_{13} \mathrm{ClO}_{6}$ \\
\hline 50 & 23.80 & 360.0772 & 359.0612 & 0.0160 & $317.2494,315.2661,245.0694,211.2592$ & Acerosin & $\mathrm{C}_{18} \mathrm{H}_{16} \mathrm{O}_{8}$ \\
\hline 51 & 24.48 & 386.2093 & 385.2741 & 0.0648 & $268.6892,176.3903,153.4738$ & Ardisinone E & $\mathrm{C}_{23} \mathrm{H}_{30} \mathrm{O}_{5}$ \\
\hline 52 & 25.02 & 318.2558 & 317.2477 & 0.0081 & $300.0236,231.3264,192.0054,178.9974,151.0025$ & Bilobol & $\mathrm{C}_{21} \mathrm{H}_{34} \mathrm{O}_{2}$ \\
\hline 53 & 25.24 & 502.1919 & 501.3211 & 0.1292 & $486.2979,473.2810,456.2825,443.2783,435.2527$ & Cycloheterophyllin & $\mathrm{C}_{30} \mathrm{H}_{30} \mathrm{O}_{7}$ \\
\hline 54 & 26.18 & 418.3083 & 417.2634 & 0.0449 & $401.6784,375.2524,335.2505,308.5951,193.0859$ & Maesaquinone & $\mathrm{C}_{26} \mathrm{C}_{42} \mathrm{H}_{42} \mathrm{O}_{4}$ \\
\hline 55 & 26.33 & 332.1551 & 331.2267 & 0.0716 & $313.2373,287.2391,254.8929,225.6182,213.1681$ & Gibberellin A4 & $\mathrm{C}_{19} \mathrm{H}_{24} \mathrm{O}_{5}$ \\
\hline 56 & 26.54 & 336.1163 & 335.1342 & 0.0179 & $332.2440,317.2474,305.2462,279.2698,230.0207$ & Berberine & $\mathrm{C}_{20} \mathrm{H}_{18} \mathrm{NO}_{4}$ \\
\hline 57 & 26.80 & 542.2879 & 541.3524 & 0.0645 & $526.3293,511.3054,493.2956,359.2590,183.6019$ & Ardisiaquinone D & $\mathrm{C}_{31} \mathrm{H}_{42} \mathrm{O}_{8}$ \\
\hline 58 & 26.93 & 426.3861 & 425.2300 & 0.1489 & $407.0769,381.0991,339.0862,257.0452,216.0410,167.2586$ & $\begin{array}{l}\text { Alpha-amyrin } \\
\text { Aln }\end{array}$ & $\begin{array}{l}311_{422} \\
\mathrm{C}_{30} \mathrm{H}_{50} \mathrm{O}\end{array}$ \\
\hline 59 & 27.05 & 528.2723 & 527.3369 & 0.0646 & $514.3290,499.3061,191.0708,165.0543,151.0386$ & Ardisiaquinone A & $\mathrm{C}_{30} \mathrm{H}_{40} \mathrm{O}_{8}$ \\
\hline 60 & 27.08 & 530.2952 & 529.3524 & 0.0572 & $514.3292,499.3058,481.2948,453.2976,347.2579,225.7453$ & Ardisiaquinone J & $\mathrm{C}_{30} \mathrm{H}_{42} \mathrm{O}_{8}$ \\
\hline 61 & 28.09 & 334.1555 & 333.2426 & 0.0871 & $279.2678,186.7317,134.0364$ & 1,5-Dibutyl methyl hydroxycitrate & $\mathrm{C}_{15} \mathrm{H}_{26} \mathrm{O}_{8}$ \\
\hline 62 & 28.37 & 496.2824 & 495.2625 & 0.0199 & $316.1812,278.8960,205.1232,181.0499,169.0134$ & Ardisenone & $\mathrm{C}_{30} \mathrm{H}_{40} \mathrm{O}_{6}$ \\
\hline
\end{tabular}




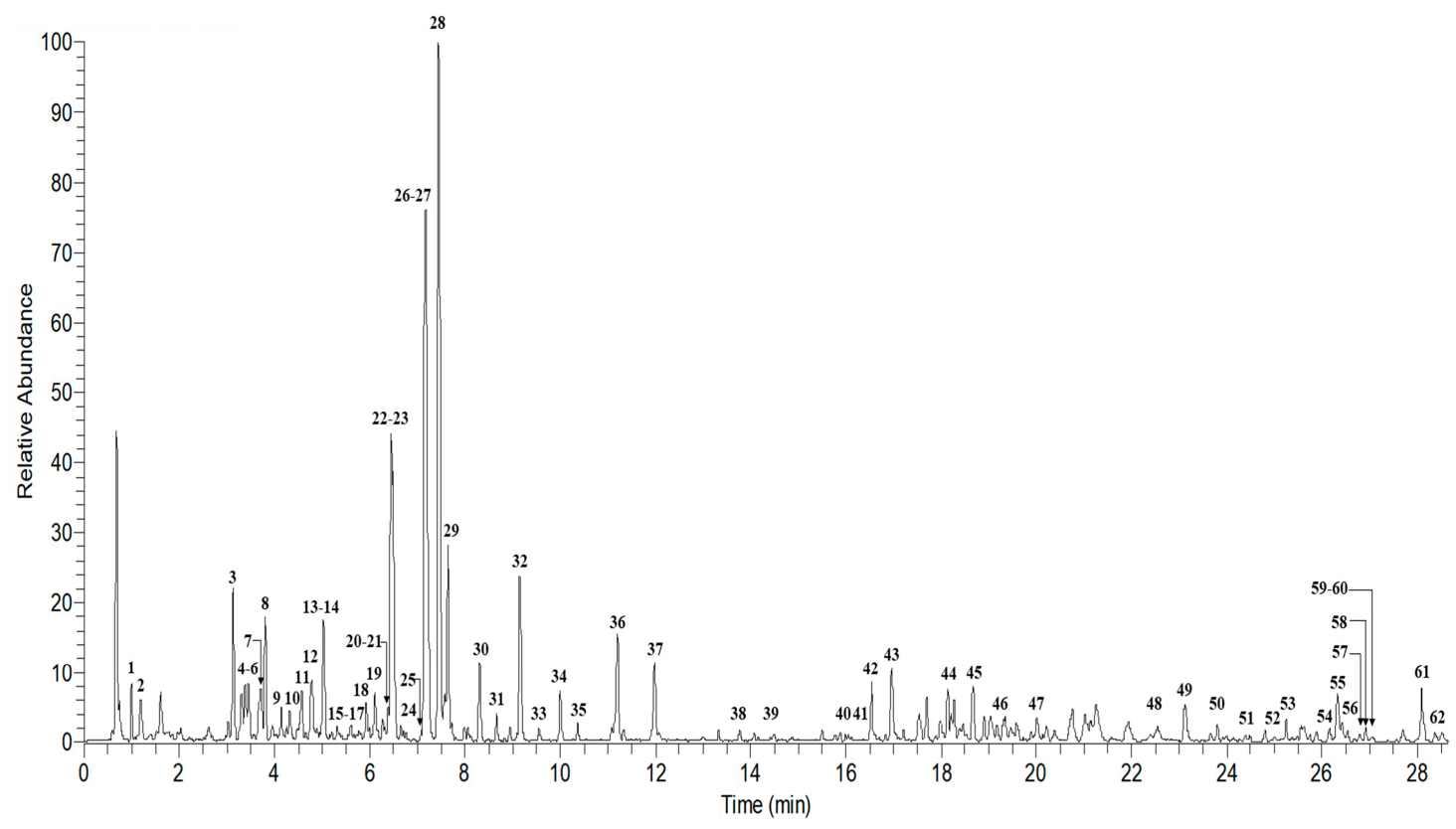

Figure 1. Total ion chromatogram of $70 \%$ ethanolic leaves extract of A. elliptica.

A total of 10 metabolites were tentatively identified as quercetin derivatives in A. elliptica leaf extracts, with the signature aglycone fragment ion at $\mathrm{m} / \mathrm{z} 301$ (peak 33) and the characteristic fragment ions at $m / z 271$ and 151 in their MS/MS spectra [20]. Peak 7 was tentatively identified as quercetin 3-O-(2"-O-galloyl)-rutinoside with a deprotonated molecule at $m / z 761.1343[\mathrm{M}-\mathrm{H}]^{-}$. Further fragmentation analysis of the compound showed fragmentation ions at $m / z 610,301$, and 169 , indicating the presence of rutin, quercetin, and gallic acid units. Peak 15 showed a deprotonated molecule at $\mathrm{m} / \mathrm{z} 755.2025$ and a fragment ion at 301, showing the loss of two rhamnosyl and one glucosyl moieties. Peak 15 was provisionally identified as quercetin 3-O-(2,6-di-O-rhamnosylglucoside) according to the comparison of previous MS/MS data [19]. Data presented in Table 2 indicated that peak 18 with $\mathrm{m} / \mathrm{z} 595.1293$ yielded fragment ion at $\mathrm{m} / \mathrm{z} 301$ attributed to the quercetin aglycone formed by the loss of a lathyrosyl residue $(294 \mathrm{u})$. Meanwhile, peak 21 was tentatively identified as rutin with a deprotonated molecule at $\mathrm{m} / \mathrm{z} 609.1450$ and gave quercetin aglycone at $\mathrm{m} / \mathrm{z} 301$ due to the loss of a rutinoside residue $(308 \mathrm{u})$. Similar to peak 23 at $\mathrm{m} / \mathrm{z} 380.9911$, loss of a sulfate residue $(80 \mathrm{u})$ yielded a fragment ion at $\mathrm{m} / \mathrm{z} 301$. Peaks $\mathbf{2 4}, \mathbf{2 5}$, and $\mathbf{2 8}$ were identified as quercetin-3-O-glucoside, quercetin-3-O-arabinoside, and quercetin-3-O-rhamnoside, based on the deprotonated molecules at $\mathrm{m} / \mathrm{z} 463.0875,433.0768$, and 447.0917, respectively. Based on the mass spectrum, the radical aglycone anion $(\mathrm{m} / \mathrm{z} 300)$ also could be observed from the regular homolytic cleavage of glycosidic bonds in the deprotonated quercetin ion $(\mathrm{m} / \mathrm{z} 301)$ by negative ion mode. The relative abundance of radical anions is affected by the collision energy with a relative increase in collision energy leading to a relative increase of radical anion [22]. Peak 20 was conditionally identified as quercetin 3-O- $\alpha$-L-rhamnoside-7-O- $\beta$-D-glucoside with a deprotonated molecule at $\mathrm{m} / \mathrm{z} 609.1451$ with fragmentation ions at $m / z 463,447$, and 301, which resulted from the removal of rhamnosyl residue $(146 \mathrm{u})$ and glucose $(162 \mathrm{u})$ moieties [19].

Peaks 16, 17, 19, and 22 were identified as myricetin derivatives based on the presence of fragmentation ion at $\mathrm{m} / \mathrm{z} 316$, which corresponds to the myricetin aglycone fragment in the MS/MS spectra [19]. Peaks 16, 17, 19, and 22 were tentatively assigned as myricetin-3-O-glucoside, myricetin-3-O-rutinoside, myricetin-3-O-arabinoside, and myricetin-3-O-rhamnoside, based on the deprotonated molecules at $m / z 479.0816,625.1396,449.0713$, and 463.0869 corresponding to the loss of sugar moieties, respectively. The radical myricetin anion $(\mathrm{m} / \mathrm{z} 316)$ observed instead of $\mathrm{m} / \mathrm{z} 317 \mathrm{might}$ be due to the scission of glycosidic bonds as a result of high collision energy in the system [22]. 
Peak 13 showed a deprotonated molecule at $m / z 289.1802$ with fragment ions at $m / z 245,179$, and 137, suggesting that the presence of catechin corresponded to the reported data [19]. Peak 3 was tentatively identified as catechin 6-C-glucoside based on deprotonated molecule at $m / z 451.3394$ and gave fragment ion at $m / z 289$ attributed to catechin aglycone formed by the loss of a glucosyl moiety (162 u). Epigallocatechin-3-gallate and its isomer were assigned as peaks 14 and 31 at $\mathrm{m} / \mathrm{z} 457.0766$ based on fragment ions observed at $m / z 305$ and $m / z 169$, which correspond to epigallocatechin and gallic acid moieties, respectively.

Peaks 26, 30, and 34 were identified as kaempferol derivatives. Peak 26 was conditionally identified as kaempferol with a deprotonated molecule at 285.0395 with the characteristic fragment ions at $m / z$ 257, 213, and 187 [19]. Peak 30 was identified as kaempferol-3-O-rhamnoside based on the removal of rhamnosyl moiety. Peak 34 was assigned as rhamnocitrin 3-O-sulfate, and fragmentations indicated that the removal of a sulfate group $(80 \mathrm{u})$ yielded a rhamnocitrin aglycone moiety $(\mathrm{m} / z 299)$, with further loss of a methyl radical (15 $\mathrm{u}$ ) giving the fragment ion at $\mathrm{m} / \mathrm{z} 285$.

Apart from quercetin, myricetin, catechin, and kaempferol derivatives, other flavonoid signals could be detected in A. elliptica leaf extract. Peak 4 was tentatively identified as 5,7-dimethoxyflavone, with a deprotonated molecule at $\mathrm{m} / \mathrm{z} 281.0331$ and fragmentation ions at $\mathrm{m} / \mathrm{z} 219$ and 201, due to the loss of two methoxy groups and one hydroxyl group. Peak 8 showed a deprotonated molecule at $\mathrm{m} / \mathrm{z} 549.1448$ and fragmentation ion at $\mathrm{m} / \mathrm{z} 269$, suggesting the presence of a formononetin unit. Peak 10 was tentatively identified as 5,2', 4', 5'-tetrahydroxy-3-(3-hydroxy-3-methylbutyl)-6", 6"-dimethylpyrano[2", $\left.3^{\prime \prime}: 7,8\right]$ flavone,

commonly named KB-2, with a deprotonated molecule at $\mathrm{m} / \mathrm{z} 453.1605$ and fragment ion at $\mathrm{m} / \mathrm{z} 367$ due to the elimination of $\mathrm{C}_{5} \mathrm{H}_{11} \mathrm{O}$ [23].

Furthermore, theasinensin A (Peak 11) could be detected with a deprotonated molecule at $\mathrm{m} / \mathrm{z}$ 913.1451 and fragment ions at $m / z 743$ and 575 due to the loss of gallic acid moieties (169u) [24]. Peaks 12, 27 , and 32 were respectively assigned as apigenin 7-sulfate, luteolin 7-sulfate, and isorhamnetin 3-sulfate (persicarin), with the elimination of a sulfate moiety $(80 \mathrm{u}$ ), yielding the apigenin fragment ion at $m / z 270$, luteolin fragment ion at $m / z 285$, and isorhamnetin fragment ion at $m / z 315[25,26]$. Several significant fragment ions were used to distinguish flavone sulfate (peak 27) from flavonol sulfate (peak 34) derivatives at $m / z$ 105, 133, and 178 which are characteristic of flavone fragmentation. Furthermore, due to the additional hydroxyl group, fragment ions at $m / z 110,151,162$, and 211 are characteristic of flavonol [27]. Peak 29 was conditionally detected as 7,2'-dihydroxyflavone 7-glucoside, with a deprotonated molecule at $m / z 415.1962$ and a fragment ion at $m / z 252$ due to the loss of a glucosyl moiety (162 u). Peak 35 was tentatively assigned as isoscutellarein 4'-methyl ether 8-(2"'-sulfatoglucoside) with the elimination of sulfate and glucosyl moieties, which resulted in fragment ions at $\mathrm{m} / \mathrm{z} 461$ and 299. Thonningianin B (peak 42) could be identified with a deprotonated molecule at $m / z 721.3632$ and fragment ion at $m / z 569$ due to the elimination of $\mathrm{C}_{7} \mathrm{H}_{4} \mathrm{O}_{4}$. Moreover, gossypetin 8-glucoside-3-sulfate, 6-chlorocatechin, acerosin, and cycloheterophyllin could also be detected in A. elliptica leaf extract, and they were assigned as peaks $45,49,50$, and 53, respectively. Interestingly, few metabolites that were reported in genus Ardisia could be tentatively identified in the currently studied leaf extract, including flavonoids, triterpenoids, benzoquinones, fatty acid derivatives, phenolic lipids, and phenol ester. Oxycoccicyanin or peonidin-3-glucoside was assigned as peak 9 with the $[\mathrm{M}-2 \mathrm{H}]^{-}$ion at $\mathrm{m} / \mathrm{z} 461.1610$ and a fragment ion at $\mathrm{m} / \mathrm{z} 300$ due to the elimination of a glucosyl moiety. Reported triterpenoids could be detected in the current leaf extract, including ardisianoside D (peak 40) with a deprotonated molecule at $\mathrm{m} / \mathrm{z} 883.4165$. The fragment ion at $\mathrm{m} / \mathrm{z} 456$ could be observed due to the loss of arabinose, xylose, and glucose moieties [28].

Furthermore, peak 46 was assigned as friedelan-3-one with a deprotonated molecule at $\mathrm{m} / \mathrm{z}$ 425.2304, consistent with reported data at $m / z 271$ and 245 due to the loss of $\mathrm{C}_{11} \mathrm{H}_{20}$ and $\mathrm{C}_{13} \mathrm{H}_{26}$, respectively [29]. Alpha-amyrin (peak 58) could also be detected based on fragment ions of a dehydrated deprotonated molecule at $m / z 407$, with residues of $\mathrm{C}_{21} \mathrm{H}_{33}, \mathrm{C}_{19} \mathrm{H}_{29}$, and $\mathrm{C}_{16} \mathrm{H}_{24}$ at $m / z 285$, 257 , and 216 respectively, which corresponded with the reported MS/MS data of alpha-amyrin [30]. 
Several benzoquinone derivatives were also tentatively identified in A. elliptica leaf extract at peaks 41, 44, 47, 54, 57, 59, and 60. Ardisianone A (peak 41) was conditionally identified with a deprotonated molecule at $m / z$ 345.1830, as well as fragmentation ions at $m / z 306$ (loss of $\left.\mathrm{C}_{3} \mathrm{H}_{7}\right), 292\left(\operatorname{loss}\right.$ of $\left.\mathrm{C}_{4} \mathrm{H}_{9}\right)$, and 192 [31]. Peak 44 showed a deprotonated molecule at $m / z 293.2114$ with fragment ions at 275 (loss of $\mathrm{H}_{2} \mathrm{O}$ ), as well as 155 and 141 (benzoquinone unit with 2 hydroxyl groups), which are characteristic of embelin [32]. Ardisiaquinones which were previously reported in Ardisia genus could be detected in the current leaf extract. Peak 47 was conditionally identified as ardisiaquinone $G$ with a deprotonated molecule at $m / z 571.2880$, as well as characteristic fragment ions at $m / z 530$ and 487 due to the loss of two acetyl units $(84 \mathrm{u})$; the fragment ion at $\mathrm{m} / \mathrm{z} 390 \mathrm{implied}$ the two fully substituted benzoquinone rings present in ardisiaquinone G [33]. Ardisiaquinone D was assigned as peak 57 with a deprotonated molecule at $m / z 541.3524$ and product ions at $m / z 526$ and 511 due to the loss of two methyl groups, while $m / z 359$ and 183 showed the presence of a benzoquinone ring. The demethylation of metabolite 57 yielded ardisiaquinone A (peak 59) with a deprotonated molecule at $m / z 527.3369$, as well as fragment ions at $m / z 514$ and 499 , due to the elimination of two methyl groups, whereas $m / z 191,165$, and 151 implied the presence of benzoquinone rings [34]. Peak 60 was identified as ardisiaquinone J with a deprotonated molecule at $m / z 529.3524$ and fragmentation patterns of $m / z 514$ (loss of methyl group), 499 (loss of methoxy group), and 347 (loss of one benzoquinone ring), which corresponded to the reported MS/MS data of ardisiaquinone J [35].

Peak 54 was assigned as maesaquinone which can be found in the Primulaceae family. Maesaquinone showed a deprotonated molecule at $\mathrm{m} / \mathrm{z} 417.2634$ with fragment ions at $\mathrm{m} / \mathrm{z} 401$ (loss of hydroxyl group), 335 (loss of $\mathrm{C}_{6} \mathrm{H}_{11}$ ), and 193, suggesting the presence of a benzoquinone ring [34]. Ardisiphenol B (peak 38) was one of the phenol esters with a deprotonated molecule at $m / z$ 375.1778 and fragmentation patterns of $\mathrm{m} / z 333$ (elimination of acetyl group) and 207 (elimination of acetyl group and $\mathrm{C}_{9} \mathrm{H}_{17}$ ), which aligned with previously reported MS/MS data of this metabolite [36]. Peak 39 was conditionally identified as ardisinol II with a deprotonated molecule at $m / z 289.1803$, as well as characteristic fragmentation ions at $m / z 245$ (loss of $\left.\mathrm{C}_{3} \mathrm{H}_{7}\right), 161,148$, and $123\left(\operatorname{loss}\right.$ of $\left.\mathrm{C}_{12} \mathrm{H}_{23}\right)$, which was consistent with reported MS/MS data [37].

Cornudentanone (peak 48) was conditionally identified with a deprotonated molecule at $\mathrm{m} / \mathrm{z}$ 377.2329 and fragmentation patterns at $m / z$ 359, 335 (loss of acetyl group), 316 (loss of ester group, $\mathrm{C}_{2} \mathrm{H}_{3} \mathrm{O}_{2}$ ), and 152, suggesting the presence of a benzoquinone ring. Peak 51 showed a deprotonated molecule at $m / z 385.2741$ and fragment ions at $m / z 268$ and 176, suggesting the presence of a hydroxyl benzoyl ion with a $\mathrm{C}_{6} \mathrm{H}_{12}$ aliphatic chain, and $m / z 153$ (presence of trihydroxyl benzoyl ion), which was then tentatively identified as ardisinone E [38]. Alkenylresorcinols such as bilobol (peak 52) could also be identified with a deprotonated molecule at $m / z 317.2477$, as well as fragment ions at $m / z 300$ (loss of hydroxyl moiety), 231 (loss of $\mathrm{C}_{6} \mathrm{H}_{13}$ aliphatic chain), 192 (loss of $\mathrm{C}_{9} \mathrm{H}_{17}$ aliphatic chain), 178, and 151, which aligned with previously reported MS/MS data [39]. Peak 62 was tentatively assigned as ardisenone with fragmentation patterns at $m / z 316$ (loss of $\mathrm{C}_{10} \mathrm{H}_{11} \mathrm{O}_{3}$ ), 278 (loss of $\mathrm{C}_{13} \mathrm{H}_{16} \mathrm{O}_{3}$ ), 205 (loss of $\mathrm{C}_{18} \mathrm{H}_{25} \mathrm{O}_{3}$ ), 181, and 169 with a deprotonated molecule at $m / z 495.2625$ [40].

Phenolic derivatives such as monogalloylglucose could be observed in A. elliptica leaf extract. Monogalloylglucose (peak 2) could be detected with a deprotonated molecule at $m / z 331.0662$ and fragment ion at $m / z 169$, suggesting the presence of a gallic acid unit due to the loss of a glucosyl moiety [19]. Peak 1 was identified with a deprotonated molecule at $m / z 191.0184$ and fragment ions at $m / z$ 173, 129, and 111 (loss of hydroxyl and carbon dioxide moieties), which are characteristic of citric acid [41]. 1,5-Dibutyl methyl hydroxycitrate (peak 61) could be identified with a deprotonated molecule at $m / z 333.2426$ and fragmentation patterns at $m / z 279$ (loss of $\mathrm{C}_{4} \mathrm{H}_{9}$ ), 186, and 134 [42]. Peak 55 showed a deprotonated molecule at $m / z 331.2267$ with fragment ions at $m / z 313$ (loss of hydroxyl moiety) and 287 (loss of $\mathrm{CHO}_{2}$ ), which was tentatively identified as Gibberellin A4. Moreover, berberine was conditionally assigned as peak 56 which showed a deprotonated molecule at $m / z 335.1342$ with fragment ions at $m / z 332,317$ (loss of hydroxyl moiety), and 279, which showed the transition of $\mathrm{C}_{20} \mathrm{H}_{18} \mathrm{NO}_{4}$ to $\mathrm{C}_{16} \mathrm{H}_{24} \mathrm{NO}_{3}$ [43]. Fatty acid derivatives could also be detected at peaks 36 and 37 with signature 
fragmentation patterns at $m / z 229,211$, and 171 [41]. With the aid of phytochemical characterization, it provides a view of the antioxidant and anti- $\alpha$-glucosidase potential of $70 \%$ ethanolic A. elliptica leaf extract. A large number of flavonoids such as quercetin, catechin, and kaempferol derivatives present in the extract were proven to play a role as antioxidant and anti- $\alpha$-glucosidase agents $[44,45]$. Other types of compounds such as triterpenoids and benzoquinones might also contribute synergistically to the overall biological activities.

\section{Materials and Methods}

\subsection{Chemicals and Reagents}

Folin-Ciocalteu reagent and absolute ethanol were purchased from Merck (Darmstadt, Germany). Sodium carbonate, DPPH, $\alpha$-glucosidase enzyme, $p$-nitrophenyl- $\alpha$-D-glucopyranose (PNPG), glycine, phosphate-buffered saline, sodium nitroprusside (SNP), and other standard compounds used, including gallic acid and quercetin, were purchased from Sigma-Aldrich Co. (St. Louis, MO, USA). LC-MS-grade methanol, purified water, formic acid (FA), and dimethyl sulfoxide (DMSO) were supplied by Fisher Scientific (Geel, Belgium).

\subsection{Plant Collection and Sample Preparation}

Leucaena leucocephala (MFI 0079/19), Muntingia calabura (SK 3345/18), Spondias dulcis (MFI 0065/19), Annona squamosa (SK 2956/16), Ardisia elliptica (MFI 0054/19), Cynometra cauliflora (SK 1757/2011), Ficus auriculata (MFI 0146/19), and Averrhoa bilimbi (MFI 0139/19) were collected from Sri Serdang, Selangor in March 2018. The plants used in this study were identified by Dr. Mohd Firdaus Ismail, an in-house botanist at the Biodiversity Unit, Institute of Bioscience, Universiti Putra Malaysia. The leaves were separated from the stem and cleaned of any impurities with a clean tissue. The leaves were subjected to air drying treatment at room temperature in the shade until a constant weight was achieved [46]. The dried leaves were then ground into fine powder using a laboratory blender (Waring Commercial, Torrington, CT, USA) and stored at $4{ }^{\circ} \mathrm{C}$ for further analysis.

\subsection{Sample Extraction}

Sample extraction was done using the method of Mediani et al. [46] with slight modification. Briefly, $10 \mathrm{~g}$ of the plant sample was weighed and soaked in $100 \mathrm{~mL}$ of $70 \%$ ethanol and subjected to sonication at a controlled temperature $\left(26-40^{\circ} \mathrm{C}\right.$ ) using a Thermo-10D Ultrasonic Cleaner (Fisher Scientific, Waltham, MA, USA) for $1 \mathrm{~h}$. The mixture was then filtered using Whatman filter paper No. 1 (GE Healthcare, Pittsburgh, PA, USA), and the crude extract was vacuum-evaporated using a rotary evaporator. The process was repeated twice using the residue of filtration to achieve maximum yield. The crude extracts were weighed and freeze-dried in a ScanVac CoolSafe Freeze dryer TM (Labogene, Lynge, Denmark). Freeze-dried samples were stored at $4{ }^{\circ} \mathrm{C}$ until further analysis.

\subsection{Total Phenolic Content (TPC) Determination}

The TPC was determined using the modified method by Zhang et al. [47]. A volume of $20 \mu \mathrm{L}$ gallic acid, which was used as the standard, was mixed with $100 \mu \mathrm{L}$ of Folin-Ciocalteu reagent in a 96-well plate. The mixture was left for $5 \mathrm{~min}$ until the addition of $80 \mu \mathrm{L}$ of $7.5 \%$ sodium carbonates to each well. The plate was then incubated in the dark at room temperature for $30 \mathrm{~min}$. The absorbance was measured at $750 \mathrm{~nm}$ using a Tecan Infinite F200 micro-plate reader (Tecan Group Ltd., Männedorf, Switzerland) in triplicate measurement. The same procedure was repeated using test samples to replace the standard. The gallic acid standard curve obtained was used to calculate the phenolic content of leaf extracts, which was expressed as mg of gallic acid equivalent per gram of crude extract (mg.GAE/g). 


\subsection{DPPH Free Radical Scavenging Assay}

The assay was done using the method of Wan et al. [48] in a 96-well plate using serial dilutions of $50 \mu \mathrm{L}$ of test sample (330-40 $\mu \mathrm{g} / \mathrm{mL})$. A volume of $100 \mu \mathrm{L}$ of DPPH was then mixed with the serial diluted test samples. Then, the mixture was incubated for $30 \mathrm{~min}$ in the dark at room temperature. The absorbance was measured at $515 \mathrm{~nm}$ using a micro-plate reader in triplicate measurement. The scavenging capacity (SC) of the leaf extract was calculated as SC $\%=\left[\left(\mathrm{A}_{0}-\mathrm{A}_{\mathrm{s}}\right) / \mathrm{A}_{0}\right] \times 100$, where $\mathrm{A}_{0}$ is the absorbance of reagent blank, whereas $A_{s}$ is the absorbance of test samples. The result was conveyed as $\mathrm{IC}_{50}$ value, signifying the concentration of sample required to scavenge $50 \%$ of $\mathrm{DPPH}$ free radicals. Quercetin (positive control) was used in this assay.

\subsection{Nitric Oxide (NO) Scavenging Assay}

Based on the method used by Tsai et al. [49], the NO scavenging assay was done on a 96-well plate. Then, $60 \mu \mathrm{L}$ of $10 \mathrm{mM}$ SNP in phosphate-buffered saline was mixed with $60 \mu \mathrm{L}$ of test samples $(330-5.16 \mu \mathrm{g} / \mathrm{mL})$ in a 96-well plate and incubated for $150 \mathrm{~min}$. Then, $60 \mu \mathrm{L}$ of freshly prepared Griess reagent was mixed with the test samples before the absorbance was measured at $550 \mathrm{~nm}$ using a micro-plate reader. Gallic acid was used as positive control, and the results were reported as $\mathrm{IC}_{50}$.

\subsection{Anti- $\alpha$-Glucosidase Assay}

Anti- $\alpha$-glucosidase assay was done as previously reported by Lee et al. [19]. The enzyme reaction was achieved using PNPG as the substrate and $\alpha$-glucosidase enzyme, which were dissolved in $50 \mathrm{mM}$ sodium phosphate buffer. Quercetin was used as the positive control. The test samples were prepared at concentrations of 500,30,25, 15,3, and $1 \mu \mathrm{g} / \mathrm{mL}$ in accordance with the preliminary data obtained through screening analysis. Then, six serial dilutions were done. Thereafter, $10 \mu \mathrm{L}$ of $\alpha$-glucosidase enzyme was pipetted into a mixture of $10 \mu \mathrm{L}$ of test sample and $130 \mu \mathrm{L}$ of $30 \mathrm{mM}$ phosphate buffer in a 96-well micro-plate. The negative control was prepared by substituting the sample with solvent, whereas blank solvent and blank sample were prepared by $140 \mu \mathrm{L}$ of $30 \mathrm{mM}$ sodium phosphate buffer with $10 \mu \mathrm{L}$ of solvent, and $140 \mu \mathrm{L}$ of $30 \mathrm{mM}$ sodium phosphate buffer with $10 \mu \mathrm{L}$ of test sample, respectively. Then, the mixture was incubated for $5 \mathrm{~min}$ at room temperature. The reaction was started by the adding $50 \mu \mathrm{L}$ of PNPG substrate into each well of test sample, as well as into the negative and positive controls, while the remaining wells received $50 \mu \mathrm{L}$ of $30 \mathrm{mM}$ sodium phosphate buffer. The mixture was then incubated for $15 \mathrm{~min}$ at room temperature. The reaction was ceased with the addition of $50 \mu \mathrm{L}$ of $2 \mathrm{M}$ glycine ( $\mathrm{pH} 10$ ). The absorbance was then measured at $405 \mathrm{~nm}$ using a micro-plate reader in triplicate measurement. The percentage inhibition of the test sample was calculated as $\%=\left[\left(a_{n}-a_{s}\right) / a_{n}\right] \times 100 \%$, where $a_{n}$ is the absorbance difference value between negative control and the blank, and $a_{s}$ is the absorbance difference value between the sample and the blank. The result was expressed as $\mathrm{IC}_{50}$ value in $\mu \mathrm{g} / \mathrm{mL}$.

\subsection{UHPLC-MS/MS Analysis}

Based on the method used by Abd Ghafar et al. [50], the UHPLC-MS/MS analysis slight adjustment was done. The UHPLC-MS/MS spectrum of the active extract was acquired using a Thermo Scientific ${ }^{\mathrm{TM}} \mathrm{Q}$ Exactive ${ }^{\mathrm{TM}}$ Hybrid Quadrupole-Orbitrap mass spectrometer equipped with an electrospray ionization (ESI) source coupled with an auto-sampler and surveyor UHPLC binary pump (Thermo Fisher Scientific, Bremen, Germany). Phytochemical separation was done using an Acquity UPLC HSS T3 column $(1.8 \mu \mathrm{m}, 2.1 \times 150 \mathrm{~mm})$. The mobile phase used in the separation was LC-MS-grade water (solvent A) and acetonitrile (solvent B), each consisting of $0.1 \%$ FA. The programmed gradient was initiated with $5 \%$ to $100 \%$ solvent B from 0.5 to $30 \mathrm{~min}$, and the solvent system was delivered at a flow rate of $0.4 \mathrm{~mL} / \mathrm{min}$. The sample was prepared in $10 \mathrm{mg} / \mathrm{mL}$ with an injection volume of $2 \mu \mathrm{L}$. Negative ion mode was done in full scan mass spectra acquisition from 150-1500 m/z with 
collision-induced dissociation (CID) energy of 30\%. The mass spectra were collected and processed using Thermo Xcalibur Qual Browser software 4.0 (Thermo Fisher Scientific Inc., Waltham, MA, USA).

\subsection{Statistical Analysis}

The results of TPC, DPPH and NO scavenging, and anti- $\alpha$-glucosidase activities were shown as means of three replicates \pm standard deviation. One-way ANOVA with Tukey's post hoc test was done to evaluate the significant effect of the factors at a confidence level of 95\%. MS Excel (Microsoft, Redmond, WA, USA) and Minitab 17 (Minitab Inc., State College, PA, USA) software was used in the statistical calculation and Pearson correlation analysis.

\section{Conclusions}

The results of the current study illustrated that Ardisia elliptica, Muntingia calabura, and Cynometra cauliflora exhibited strong antioxidant and anti- $\alpha$-glucosidase activities. A. elliptica showed the most potent activity among all tested plants. A total of 62 metabolites were tentatively characterized in $A$. elliptica $70 \%$ ethanolic leaf extract including flavonoids, benzoquinones, and triterpenoids which might contribute to the significant biological activities. To the best of our knowledge, this study provides the first detailed metabolite profile of $A$. elliptica by using UHPLC-ESI-Orbitrap MS. The findings in this work suggest that the leaves of A. elliptica could serve as a potential natural source of antioxidant and anti-diabetic agents. However, extensive studies are required to examine the safety and efficacy of their pharmacological properties for the utilization of alternative remedies for disease, particularly diabetes.

Author Contributions: P.L.W. and N.A.F., writing —original draft preparation, plant collection, data collection, and experimentation; S.N.M.Y., plant collection, data collection, and methodology; N.A.A.H., investigation and methodology; S.Z.A.G., data curation and methodology; A.A., methodology and statistical analysis; N.K.Z.Z., validation and methodology; F.A., conceptualization, project administration, reviewing and editing, and supervision. All authors have read and agreed to the published version of the manuscript.

Funding: This research was supported by a grant from Universiti Putra Malaysia (UPM/700/2/1/GPB/2017/9597400) under a Putra High Impact Grant Scheme.

Acknowledgments: The authors wish to thank Universiti Putra Malaysia for the facilities. The first author also gratefully acknowledges the support from Universiti Putra Malaysia for funding her study under the Graduate Research Fellowship scheme.

Conflicts of Interest: The authors have no conflicts of interest to disclose.

\section{References}

1. IPGRI. Neglected and Underutilized Plant Species: Strategic Action Plan of the International Plant Genetic Resources Institute; International Plant Genetic Resources Institute: Rome, Italy, 2002; pp. 1-30.

2. Feisul, M.I.; Azmi, S. National diabetes registry report. Minist. Health Malays. 2013, 1, 2009-2012.

3. Trigwell, S.M.; Radford, P.M.; Page, S.R. Islet glutamic acid decarboxylase modified by reactive oxygen species is recognized by antibodies from patients with type 1 diabetes mellitus. Clin. Exp. Immunol. 2001, 126, 242-249. [CrossRef] [PubMed]

4. Bouayed, J.; Bohn, T. Exogenous antioxidants-Double-edged swords in cellular redox state: Health beneficial effects at physiologic doses versus deleterious effects at high doses. Oxid. Med. Cell Longev. 2010, 3, 228-237. [CrossRef] [PubMed]

5. Kim, K.Y.; Nam, K.A.; Kurihara, H.; Kim, S.M. Potent $\alpha$-glucosidase inhibitors purified from the red alga Grateloupia elliptica. Phytochemistry 2008, 69, 2820-2825. [CrossRef] [PubMed]

6. Triggle, C.R.; Ding, H. Cardiovascular impact of drugs used in the treatment of diabetes. Ther. Adv. Chronic Dis. 2014, 5, 245-268. [CrossRef] [PubMed]

7. Kumar, S.; Narwal, S.; Kumar, V.; Prakash, O. $\alpha$-glucosidase inhibitors from plants: A natural approach to treat diabetes. Pharmacogn. Rev. 2011, 5, 19-29. [CrossRef]

8. Rahman, M.M.; Khan, F.E.; Das, R.; Hossain, M.A. Antioxidant activity and total phenolic content of some indigenous fruits of Bangladesh. Int. Food Res. J. 2016, 23, 2399-2404. 
9. Do, Q.D.; Angkawijaya, A.E.; Tran-Nguyen, P.L.; Huynh, L.H.; Soetaredjo, F.E.; Ismadji, S.; Ju, Y.H. Effect of extraction solvent on total phenol content, total flavonoid content, and antioxidant activity of Limnophila aromatica. J. Food Drug Anal. 2014, 22, 296-302. [CrossRef]

10. Mariod, A.A.; Abdelwahab, S.I.; Elkheir, S.; Ahmed, J.M.; Fauzi, P.N.M.; Chuen, C.S. Antioxidant activity of different parts from Annona squamosa, and Catunaregam nilotica methanolic extract. Acta Sci. Pol. Technol. Aliment. 2012, 11, 249-257.

11. Rabeta, M.S.; Nur Faraniza, R. Total phenolic content and ferric reducing antioxidant power of the leaves and fruits of Garcinia atrovirdis and Cynometra cauliflora. Int. Food Res. J. 2013, 20, 1691-1696.

12. Zolkeflee, N.K.Z.; Isamail, N.A.; Maulidiani, M.; Abdul Hamid, N.A.; Ramli, N.S.; Azlan, A.; Abas, F. Metabolite variations and antioxidant activity of Muntingia calabura leaves in response to different drying methods and ethanol ratios elucidated by NMR-based metabolomics. Phytochem. Anal. 2020, in press, 1-15. [CrossRef]

13. Al-Abd, N.M.; Nor, Z.M.; Mansor, M.; Zajmi, A.; Hasan, M.S.; Azhar, F.; Kassim, M. Phytochemical constituents, antioxidant and antibacterial activities of methanolic extract of Ardisia elliptica. Asian Pac. J. Trop. Biomed. 2017, 7, 569-576. [CrossRef]

14. Rahardhian, M.R.R.; Murti, B.T.; Wigati, D.; Suharsanti, R.; Putri, C.N. Solvent concentration effect on total flavonoid and total phenolic contents of Averrhoa bilimbi leaf extract. Pharmaciana 2019, 9, 137-144. [CrossRef]

15. Sampaio, B.L.; Edrada-Ebel, R.; Da Costa, F.B. Effect of the environment on the secondary metabolic profile of Tithonia diversifolia: A model for environmental metabolomics of plants. Sci. Rep. 2016, 6, 1-11. [CrossRef] [PubMed]

16. Liang, N.; Kitts, D. Antioxidant property of coffee components: Assessment of methods that define mechanism of action. Molecules 2014, 19, 19180-19208. [CrossRef]

17. Sumanont, Y.; Murakami, Y.; Tohda, M.; Vajragupta, O.; Matsumoto, K.; Watanabe, H. Evaluation of the nitric oxide radical scavenging activity of manganese complexes of curcumin and its derivative. Biol. Pharm. Bull. 2004, 27, 170-173. [CrossRef]

18. Ado, M.A.; Abas, F.; Ismail, I.S.; Ghazali, H.M.; Shaari, K. Chemical profile and antiacetylcholinesterase, antityrosinase, antioxidant and $\alpha$-glucosidase inhibitory activity of Cynometra cauliflora L. leaves. J. Sci. Food Agric. 2014, 95, 635-642. [CrossRef]

19. Lee, S.Y.; Mediani, A.; Ismail, I.S.; Maulidiani; Abas, F. Antioxidants and $\alpha$-glucosidase inhibitors from Neptunia oleracea fractions using ${ }^{1} \mathrm{H}$ NMR-based metabolomics approach and UHPLC-MS/MS analysis. BMC Complem. Altern. Med. 2019, 19, 1-15. [CrossRef]

20. Gavillán-Suárez, J.; Aguilar-Perez, A.; Rivera-Ortiz, N.; Rodríguez-Tirado, K.; Figueroa-Cuilan, W.; Morales-Santiago, L.; Martínez-Montemayor, G.; Cubano, L.A.; Martínez-Montemayor, M.M. Chemical profile and in vivo hypoglycemic effects of Syzygium jambos, Costus speciosus and Tapeinochilos ananassae plant extracts used as diabetes adjuvants in Puerto Rico. BMC Complem. Altern. Med. 2015, 15, 1-15. [CrossRef]

21. Abdul-Hamid, N.A.; Maulidiani, M.; Mediani, A.; Yahya, U.I.I.; Ismail, I.S.; Tham, C.L.; Shadid, K.; Abas, F. Physicochemical characteristics, nutritional composition, and phytochemical profiles of nine Algerian date palm fruit (Phoenix dactylifera L.) varieties. J. Food Biochem. 2018, 42, e12663. [CrossRef]

22. Hvattum, E.; Ekeberg, D. Study of the collision-induced radical cleavage of flavonoid glycosides using negative electrospray ionization tandem quadrupole mass spectrometry. J. Mass Spectrom. 2003, 38, 43-49. [CrossRef]

23. Fujimoto, Y.; Zhang, X.; Kirisawa, M.; Uzawa, J.; Sumatra, M. New flavones from Artocarpus communis forst. Chem. Pharm. Bull. 1990, 38, 1787-1789. [CrossRef]

24. Hatano, T.; Kusuda, M.; Hori, M.; Shiota, S.; Tsuchiya, T.; Yoshida, T. Theasinensin A, a tea polyphenol formed from (-)-epigallocatechin gallate, suppresses antibiotic resistance of methicillin-resistant Staphylococcus aureus. Planta Med. 2003, 69, 984-989. [CrossRef] [PubMed]

25. Kwak, J.Y.; Seok, J.K.; Suh, H.J.; Choi, Y.H.; Hong, S.S.; Kim, D.S.; Boo, Y.C. Anti-melanogenic effects of luteolin 7-sulfate isolated from Phyllospadix iwatensis Makino. Brit. J. Dermatol. 2016, 175, 501-511. [CrossRef] [PubMed]

26. Teles, Y.C.F.; Souza, M.S.R. Sulphated flavonoids: Biosynthesis, structures, and biological activities. Molecules 2018, 23, 480. [CrossRef]

27. Cuyckens, F.; Ma, Y.L.; Pocsfalvi, G.; Claeysi, M. Tandem mass spectral strategies for the structural characterization of flavonoid glycosides. Analusis 2000, 28, 888-895. [CrossRef] 
28. Chang, X.; Li, W.; Jia, Z.; Satou, T.; Fushiya, S.; Koike, K. Biologically active triterpenoid saponins from Ardisia japonica. J. Nat. Prod. 2007, 70, 179-187. [CrossRef] [PubMed]

29. Hisham, A.; Jayakumar, G.; Fujimoto, Y.; Hara, N. 1 $\beta, 15 \alpha$-dihydroxyfriedelan-3-one, a triterpene from Salacia beddomei. Phytochemistry 1996, 43, 843-845. [CrossRef]

30. Naumoska, K.; Vovk, I. Analysis of triterpenoids and phytosterols in vegetables by thin-layer chromatography coupled to tandem mass spectrometry. J. Chromatogr. A 2015, 1381, 229-238. [CrossRef]

31. Fukuyama, Y.; Kiriyama, Y.; Okino, J.; Kodama, M.; Iwaki, H.; Hosozawa, S.; Matsui, K. Naturally occuring 5-lipoxygenase inhibitor. II. Structures and syntheses of ardisianones A and B, and maesanin, alkenyl-1,4-benzoquinones from the rhizome of Ardisia japonica. Chem. Pharm. Bull. 1993, 41, 561-565. [CrossRef] [PubMed]

32. Joy, B.; Sivadasan, R.; Emilia, A.T.; John, M.; Sobhan, P.K.; Seervi, M.; Santhoshkumar, T.R. Lysosomal destabilization and Cathepsin B contributes for cytochrome $\mathrm{c}$ release and caspase activation in embelin-induced apoptosis. Mol. Carcinogen. 2010, 49, 324-336. [CrossRef]

33. Yang, L.; Khoo-beattie, C.; Goh, K.; Chng, B.; Yoganathan, K.; Lai, Y.; Butler, M.S. Ardisiaquinones from Ardisia teysmanniana. Phytochemistry 2001, 58, 1235-1238. [CrossRef]

34. Ogawa, H.; Natori, S. Hydroxybenzoquinones from Myrsinaceae plants. The structures of 2-hydroxy-5-methoxy-3-pentadecenylbenzoquinone and ardisiaquinones A, B and C from Ardisia spp. Chem. Pharm. Bull. 1968, 16, 1709-1720. [CrossRef]

35. Ndonsta, B.L.; Tatsimo, J.S.N.; Csupor, D.; Forgo, P.; Berkecz, R.; Berenyi, A.; Tene, M.; Molnar, J.; Zupko, I.; Hohmann, J.; et al. Alkylbenzoquinones with antiproliferative effect against human cancer cell lines from stem of Ardisia kivuensis. Phytochem. Lett. 2011, 4, 227-230. [CrossRef]

36. Sumino, M.; Sekine, T.; Ruangrungsi, N.; Ikegami, F. Ardisiphenols A-C, novel antioxidants from the fruits of Ardisia colorata. Chem. Pharm. Bull. 2001, 49, 1664-1665. [CrossRef]

37. Sun, W.Y.; Zong, Q.; Gu, R.L.; Pan, B.C. Synthesis of ardisinol II. Synthesis 1998, 11, 1619-1622. [CrossRef]

38. Zheng, Y.; Deng, Y.; Wu, F. Ardisinones A-E, novel diarylundecanones from Ardisia arborescens. J. Nat. Prod. 2004, 67, 1617-1619. [CrossRef]

39. Ndontsa, B.L.; Dongmo, F.L.M.; Tala, M.F.; Wabo, K.; Zeng, G.; Tan, N.; Tane, P. A new cytotoxic alkenylresorcinol from Embelia schimperi. Rec. Nat. Prod. 2013, 8, 37-40.

40. David Horgen, F.; Guinaudeau, H.; Pezzuto, J.M.; Soejarto, D.D.; Farnsworth, N.R. Isolation and structure elucidation of ardisenone: A new, cytotoxic alkenylphenol from Ardisia iwahigensis. J. Nat. Prod. 1997, 60, 533-535. [CrossRef]

41. Taamalli, A.; Arráez-Román, D.; Abaza, L.; Iswaldi, I.; Fernández-Gutiérrez, A.; Zarrouk, M.; Segura-Carretero, A. LC-MS-based metabolite profiling of methanolic extracts from the medicinal and aromatic species Mentha pulegium and Origanum majorana. Phytochem. Analysis 2015, 26, 320-330. [CrossRef]

42. Mackeen, M.M.; Mooi, L.Y.; Amran, M.; Mat, N.; Lajis, N.H.; Ali, A.M. Noncytotoxic and antitumour-promoting activities of garcinia acid esters from Garcinia atroviridis Griff. ex T. Anders (Guttiferae). Evid. Based Compl. Alt. 2012, 2012, 1-5. [CrossRef]

43. Scazzocchio, F.; Cometa, M.F.; Tomassini, L.; Palmery, M. Antibacterial activity of Hydrastis canadensis extract and its major isolated alkaloids. Planta Med. 2001, 67, 561-564. [CrossRef]

44. Li, Y.Q.; Zhou, F.C.; Gao, F.; Bian, J.S.; Shan, F. Comparative evaluation of quercetin, isoquercetin and rutin as inhibitors of $\alpha$-Glucosidase. J. Agric. Food Chem. 2009, 57, 11463-11468. [CrossRef]

45. Atoui, A.K.; Mansouri, A.; Boskou, G.; Kefalas, P. Tea and herbal infusions: Their antioxidant activity and phenolic profile. Food Chem. 2005, 89, 27-36. [CrossRef]

46. Mediani, A.; Abas, F.; Tan, C.; Khatib, A. Effects of different drying methods and storage time on free radical scavenging activity and total phenolic content of Cosmos Caudatus. Antioxidants 2014, 3, 358-370. [CrossRef]

47. Zhang, Q.; Zhang, J.; Shen, J.; Silva, A.; Dennis, D.A.; Barrow, C.J. A simple 96-well microplate method for estimation of total polyphenol content in seaweeds. J. Appl. Phycol. 2006, 18, 445-450. [CrossRef]

48. Wan, C.; Yuan, T.; Cirello, A.L.; Seeram, N.P. Antioxidant and $\alpha$-glucosidase inhibitory phenolics isolated from highbush blueberry flowers. Food Chem. 2012, 135, 1929-1937. [CrossRef] 
49. Tsai, P.; Tsai, T.; Yu, C.; Ho, S. Comparison of NO-scavenging and NO-suppressing activities of different herbal teas with those of green tea. Food Chem. 2007, 103, 181-187. [CrossRef]

50. Abd Ghafar, S.Z.; Mediani, A.; Maulidiani; Ramli, N.Z.; Abas, F. Antioxidant, $\alpha$-glucosidase, and nitric oxide inhibitory activities of Phyllanthus acidus and LC-MS/MS profile of the active extract. Food Bioscience 2018, 25, 134-140. [CrossRef]

Sample Availability: Samples of the compounds are available from the authors.

(C) 2020 by the authors. Licensee MDPI, Basel, Switzerland. This article is an open access article distributed under the terms and conditions of the Creative Commons Attribution (CC BY) license (http://creativecommons.org/licenses/by/4.0/). 